\title{
Estimation Method of Maximum Inter-Story Drift Angle of Wood-Frame House using Two Accelerometers
}

\author{
Ryusei Fukuzawa ${ }^{1, a,{ }^{*},}$, Akira Mita ${ }^{1, b}$, Tomonori Murakami ${ }^{2, c}$ \\ 'Department of System Design Engineering, Keio University, Tokyo, Japan \\ ${ }^{2}$ Mitsui Home Co., Ltd. Technology Research \& Development Institute, Tokyo, Japan \\ a ryu.fukufuku99@keio.jp, b mita@sd.keio.ac.jp, ct-murakami@mitsuihome.co.jp
}

Keywords: Structural Health Monitoring, Maximum Inter-Story Drift Angle, Wood-Frame Construction

\begin{abstract}
In April 2016, Kumamoto earthquake occurred in Japan and many wooden houses collapsed and many lives were lost because of the second and larger main shock. As a result, the need for Structural Health Monitoring (SHM) for wooden houses is receiving increased attention. In the SHM system, maximum inter-story drift angle is considered as the damage index. We assume that the first story of a wooden house will be damaged so that we need only to focus on the response of this first story. Hence, we install accelerometers on the ground floor and the second floor. In order to estimate the inter-story drift angle, we need to integrate the acceleration records twice. The simple double integration will result in erroneous results. Thus, in this paper, we propose the most appropriate integration method to estimate the maximum story drift angle with high accuracy using two accelerometers.
\end{abstract}

\section{Introduction}

Structural Health Monitoring

In April 2016, Kumamoto earthquake occurred in Japan and many wooden houses collapsed and many lives were lost. The significant feature of this earthquake was that there were two main shocks and the latter was larger. Thus several houses which survived during the first main shock collapsed during the second main shock resulting in a few casualties as they came back home. This incident reaffirmed the importance and the usefulness of Structural Health Monitoring (SHM) for wooden houses. In the SHM system, maximum inter-story drift angle is considered as the damage index. We assume that the first story of a wooden house will collapse so that we will only focus on the response of this first story. Hence, we install accelerometers on the ground floor and the ceiling of the ground floor. In order to estimate the inter-story drift angle from the sensors, we have to integrate the acceleration records twice. The simple second-order integration without a careful numerical consideration will result in erroneous results. Thus, in this paper, we tried several approaches to propose the most appropriate integration method to estimate the maximum story drift angle with high accuracy using two accelerometers.

\section{Previous researches and their problems}

There is very limited research on SHM for wooden houses. The previous research ${ }^{[1][2]}$ has focused on simple vibration models and steel-frame buildings. In order to implement SHM systems into wooden houses, it is useful to perform seismic analysis for wooden houses. In addition, it is important to analyze the response characteristics of buildings by vibrating with 
various types of seismic waves because the response of the buildings vary greatly depending on the type of seismic waves.

The purpose of this research

In general, it is difficult to obtain accurate displacement time histories from acceleration data due to several sources of noise. This paper aims to establish a method for estimating the maximum inter-story drift angle by analyzing the acceleration data measured in full-scale shaking table tests of a wood-frame house. In the test, we used low-cost MEMS type acceleration sensors along with high-precision sensors to seek the possibility to reduce the system cost.

\section{Proposed method}

In this study, we estimate the maximum inter-story drift angle from acceleration data acquired by two types of acceleration sensors using the following three methods. In SHM system, displacement data are often calculated from acceleration sensors installed at the base or inside of the building. We can obtain the relative response displacement by integrating the acceleration data of the base and each floor. However, the measured acceleration data contain small errors due to noise and baseline misalignment of the acceleration sensors. Although these errors are small, these errors are dramatically amplified thorough second-order integration and the displacement tends to diverge. There are two methods for calculating displacement from acceleration: time domain integration and frequency domain integration.

\section{(Method1) Iwan's method}

Iwan's method ${ }^{[2][3]}$ is a method to compensate for the acceleration baseline misalignment in the time domain. In this method, the errors in integration are assumed to be derived from the nonlinearity of the accelerometer and acceleration records are classified into three regions as shown in Fig. 1.

\section{Region1}

Region 1 is defined as a region where the maximum acceleration does not exceed 50 gal from the first point in the acceleration record. The initial baseline misalignment is assumed to be the average of the acceleration records in region 1.

$$
a_{B L}=\frac{1}{N} \sum_{i=1}^{N} a_{i}
$$

Where, $a_{B L}$ is initial baseline misalignment, $N$ is the number of acceleration data in the region 1 and $a_{i}$ is acceleration record in the region 1.

II. Region2

Region 2 is defined as a region between region1 and region3. In this region, we calculate the baseline misalignment by finding a value such that the velocity is connected to region3.

III. Region3

Region 3 is defined as a region where the maximum acceleration does not exceed 50 gal from the last point in the acceleration record. As in region1, the baseline misalignment is 
assumed to be constant. In this case, it is calculated by first approximation in the region 3 after integrating the acceleration records and obtaining the velocity records.

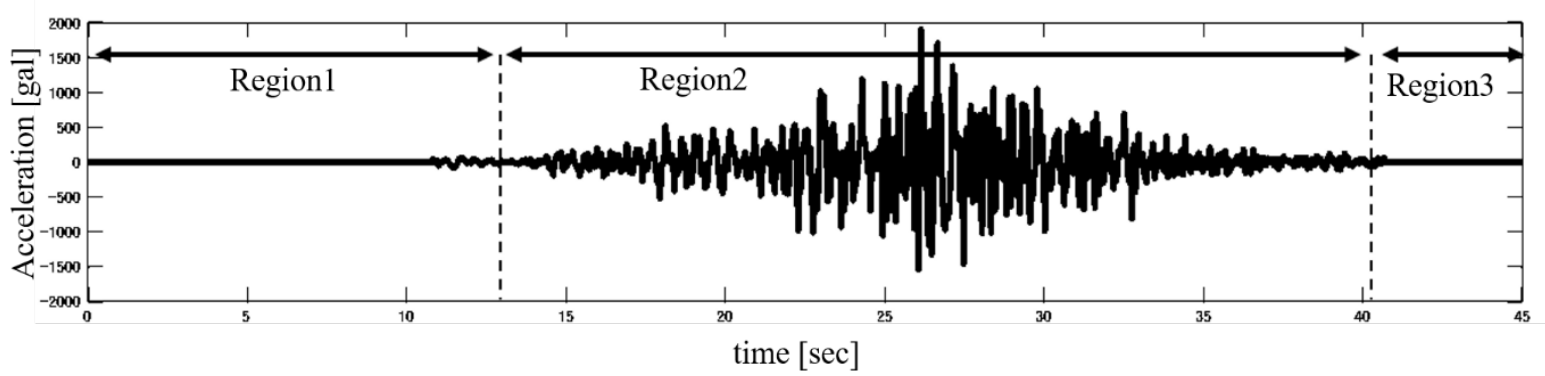

Fig.1 Classification of acceleration record

(Method2) Method of applying a window function to acceleration record

In this study, a window function was applied to the acceleration records and the stepwise errors in the acceleration records were corrected by cutting out the main motion. The window function is often used in Fourier analysis and audio compression because it is a function that sets the signals outside the finite interval to zero so that only the signals within the finite interval are cut out. The accuracy of estimating the maximum inter-story drift angle is verified by applying the Hamming window as follows.

$$
w_{r}(n)=\left\{\begin{array}{c}
0.54-0.46 \cos \left(\frac{2 \pi n}{N-1}\right)(0 \leq n<N-1) \\
0 \quad(n \geq N)
\end{array}\right.
$$

(Method3) Method for calculating the displacement using only the real part of the displacement Fourier spectrum

We focused on the possibility that high-pass filtering on acceleration records may distort significant data and the maximum story drift angle was estimated using only the real part of the displacement Fourier spectrum. Even when residual displacement occurs in a building, the displacement can be calculated from the real component of the displacement Fourier spectrum.

$$
D(\omega)=\int_{-\infty}^{\infty} d(t) \cdot e^{-j \omega t} d t
$$

Where, $d(t)$ is displacement with residual displacement $d_{\infty}$ and $D(\omega)$ is frequency function of $d(t)$. Moreover, assuming that the time function $d(t)$ is a casual function, the real part $D_{e}(\omega)$ and the imaginary part $D_{o}(\omega)$ of the frequency function $\mathrm{D}(\omega)\left(=D_{e}(\omega)+j D_{o}(\omega)\right)$ are in a Hilbert transform relationship as follows.

$$
D_{o}(\omega)=-\frac{1}{\pi} \int_{-\infty}^{\infty} \frac{D_{e}(s)}{\omega-s} d s
$$




$$
D_{e}(\omega)=\frac{1}{\pi} \int_{-\infty}^{\infty} \frac{D_{o}(s)}{\omega-s} d s
$$

The sign function $\operatorname{sgn}(t)$ is defined as Eq.(6) and (7).

$$
\begin{gathered}
\operatorname{sgn}(t)=-1(\mathrm{t}<0), 0(\mathrm{t}=0), 1(\mathrm{t}>0) \\
\operatorname{sgn}(t) \rightarrow \frac{2}{\mathrm{j} \omega}
\end{gathered}
$$

Using the sign function $\operatorname{sgn}(t)$, the unit step function $U(t)$ is written as Eq.(8).

$$
U(t)=\frac{1+\operatorname{sgn}(t)}{2}
$$

Using the unit step function $U(t)$, the time function $d(t)$ is written as Eq.(9).

$$
d(t)=2 U(t) \cdot d_{e}(t)=2 U(t) \cdot d_{o}(t)
$$

Eq.(9) shows that the time function $d(t)$ of the displacement can be calculated from only the real part $D_{e}(\omega)$ or the imaginary part $D_{o}(\omega)$ of the frequency function $\mathrm{D}(\omega)$. On the other hand, if the time function $d(t)$ has a residual displacement $d_{\infty}\left(=\lim _{t \rightarrow \infty} d(t) \neq 0\right)$, it can be separated into a time limiting function $d_{T L}(t)$ and a unit step function $U(t)$ satisfying Eq.(4) and (5).

$$
d(t)=d_{T L}(t)+d_{\infty} \cdot U(t)
$$

Eq.(11) can be obtained by Fourier transforming Eq.(10) and the second term is a generalized function with a singularity at $\omega=0$.

$$
D(\omega)=D_{T L}(\omega)+d_{\infty} \cdot\left(\pi \delta(\omega)+\frac{1}{j \omega}\right)
$$

As well as the time limiting function $D_{T L}(\omega)$, the time function of the displacement $d(t)$ can be calculated from only the real or imaginary part of the frequency function in the case of residual displacement.

\section{Estimation results}

A full-scale shaking table test

The test house was three-story wooden house with two-by-four method designed by Mitsui Home Corp. The following figures show the south and north elevation. Strain gauge type (AS10GB) and low-cost MEMS type (LIS3DSH) of accelerometers were used to verify the accuracy of estimating the maximum inter-story drift angle of the first floor by each accelerometer. 


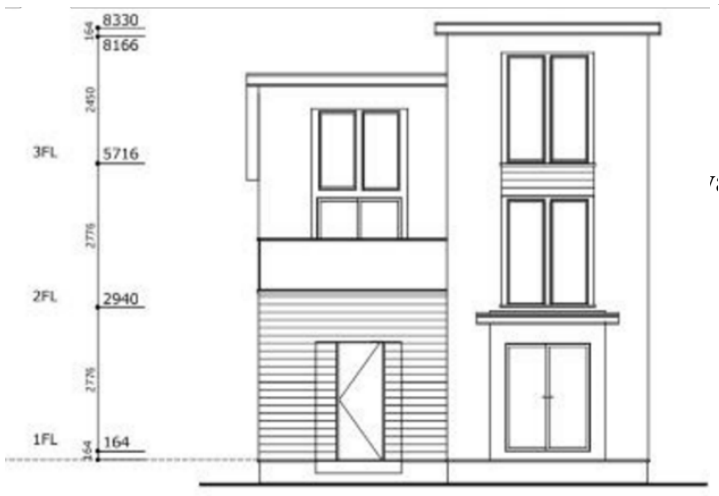

SOUTH

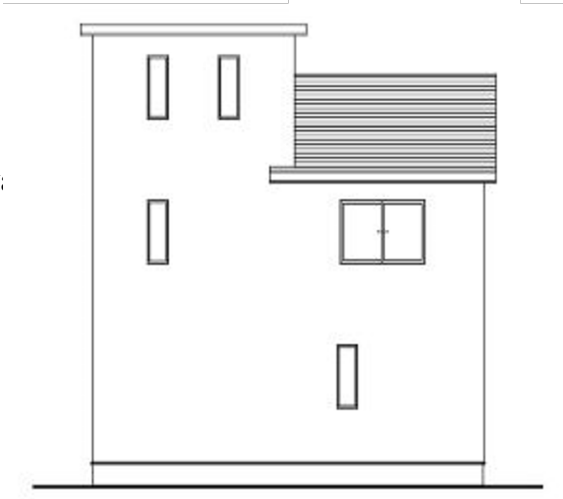

NORTH

Fig. 2 Elevation of the building

In this shaking table test, several types of earthquake waves were used for excitation. First, Kumamoto wave was used for 120 times and after that, other waves were used once. Table 1 shows the list of earthquake waves.

Table. 1 Seismic waves

\begin{tabular}{|l|c|c|}
\hline \multicolumn{1}{|c|}{ Earthquake } & Input scale & Number of times \\
\hline \hline Kumamoto & $100 \%$ & 120 \\
\hline JMA Kobe & $100 \%$ & 1 \\
\hline Shiogama & $100 \%$ & 1 \\
\hline Tottori & $100 \%$ & 1 \\
\hline Nankai Trough & $100 \%$ & 1 \\
\hline
\end{tabular}

Estimation accuracy of the maximum inter-story drift angle

Table 3 shows the error of the maximum inter-story drift angle calculated by directly integrating the acceleration record(Before correction) and using the above 3 methods. The error in Table 3 represents the error for the true value of the maximum inter-story drift angle calculated from displacement measurement in the shaking table test as shown in Table2.

Table.2 True Value of the maximum inter-story drift angle

\begin{tabular}{|l|c|}
\hline \multicolumn{1}{|c|}{ Earthquake } & Maximum inter-story drift angle [rad] \\
\hline \hline Kumamoto & $1 / 108$ \\
\hline JMA Kobe & $1 / 91$ \\
\hline Shiogama & $1 / 59$ \\
\hline Tottori & $1 / 40$ \\
\hline Nankai Trough & $1 / 23$ \\
\hline
\end{tabular}


Table.3 The error for each method

\begin{tabular}{|l|c|c|c|c|}
\hline \multicolumn{1}{|c|}{ Earthquake } & $\begin{array}{c}\text { Before } \\
\text { correction }\end{array}$ & Method1 & Method2 & Method3 \\
\hline \hline Kumamoto & $4.2 \%$ & $42.4 \%$ & $2.1 \%$ & $4.2 \%$ \\
\hline JMA Kobe & $26.5 \%$ & $4.4 \%$ & $8.2 \%$ & $11.8 \%$ \\
\hline Shiogama & $12.4 \%$ & $5.9 \%$ & $5.9 \%$ & $5.9 \%$ \\
\hline Tottori & $6.2 \%$ & $4.9 \%$ & $4.0 \%$ & $2.0 \%$ \\
\hline Nankai Trough & $6.4 \%$ & $1.6 \%$ & $2.3 \%$ & $2.3 \%$ \\
\hline
\end{tabular}

In method1, the error is worse in Kumamoto indicating that the correction accuracy is sensitive depending on the type of seismic wave. On the other hand, method 2 and method3 improved the estimation accuracy of the maximum inter-story drift angle for all seismic waves. The error of both methods is within $12 \%$, which enables us to estimate the maximum inter-story drift angle stably regardless of the type of earthquake. A possible error factor is the difference in the characteristics of each seismic waves. As shown in Fig.3, acceleration response spectrum of Kumamoto and JMA Kobe which has a relatively large error shows that response acceleration around $\mathrm{T}=0.2[\mathrm{~s}]$ which is the natural period of the building is small. In other words, the error becomes larger for seismic waves with a small $\mathrm{S} / \mathrm{N}$ ratio around the first natural frequency of the building.
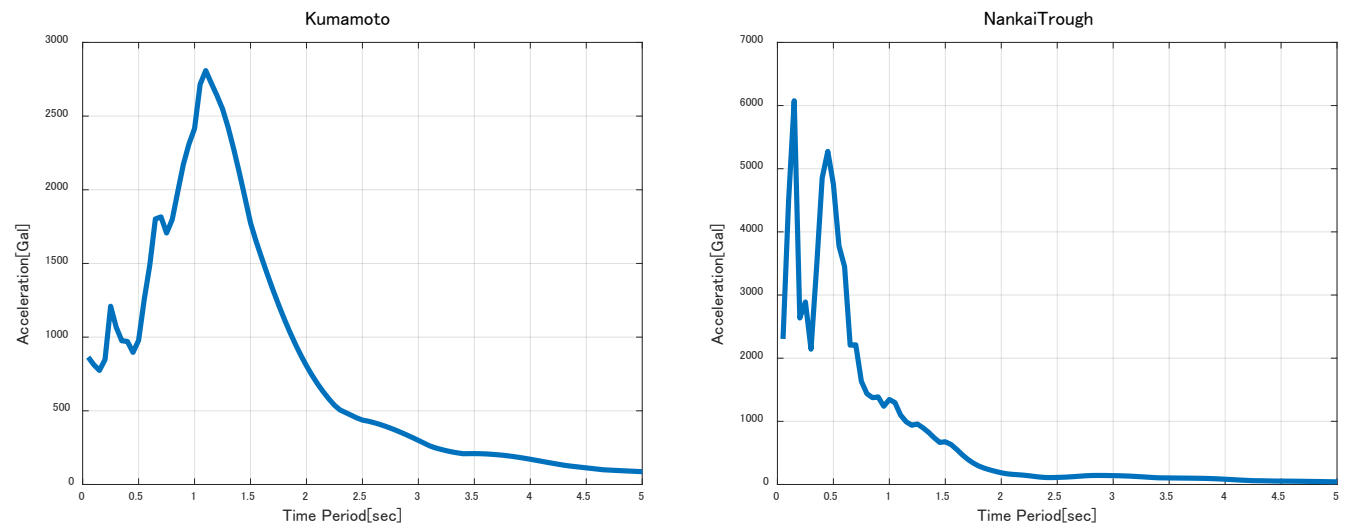

Fig.3 Acceleration response spectrum

\section{Consideration of accelerometers}

As mentioned above, two types of acceleration sensors were used to measure the acceleration data in this shaking table test. The error of the maximum inter-story drift angle estimated by each accelerometer was compared considering the instrumental characteristics of each accelerometer. The error in Table4 represents the error for the true value of the maximum inter-story drift angle calculated from displacement measurement in the shaking table test as shown in Table2. 
Table.4 The error for each accelerometer

\begin{tabular}{|c|c|c|c|c|}
\hline \multirow{2}{*}{ Earthquake } & \multicolumn{2}{|c|}{ Method2 } & \multicolumn{2}{c|}{ Method3 } \\
\cline { 2 - 5 } & Strain-gauge & MEMS & Strain-gauge & MEMS \\
\hline \hline Kumamoto & $2.1 \%$ & $6.3 \%$ & $4.2 \%$ & $6.2 \%$ \\
\hline JMA Kobe & $8.2 \%$ & $4.7 \%$ & $11.8 \%$ & $14.5 \%$ \\
\hline Shiogama & $5.9 \%$ & $11.8 \%$ & $5.9 \%$ & $9.4 \%$ \\
\hline Tottori & $4.0 \%$ & $8.0 \%$ & $2.0 \%$ & 4.0 \\
\hline Nankai Trough & $2.3 \%$ & $2.3 \%$ & $2.3 \%$ & $4.5 \%$ \\
\hline
\end{tabular}

Both acceleration sensors were able to estimate the maximum inter-story drift angle for all types of seismic waves within $15 \%$. In other words, this study suggests the possibility of lowcost MEMS type accelerometer for SHM system.

\section{Conclusion}

In this paper, we proposed and compared 3 methods to estimate the maximum inter-story drift angle using accelerometers installed on the first and second floors of a wood frame house. While the estimation accuracy of Iwan's method was not stable, the method of applying a window function to acceleration record and the method for calculating the displacement using only the real part of the displacement Fourier spectrum were able to estimate the maximum inter-story drift angle for all types of seismic data within an error rate of $15 \%$. In addition, the latter method can also be used to evaluate the residual displacement that occurred in a building after an earthquake. It was also suggested that low-cost MEMS type accelerometers can be used for SHM as well as strain gauge type of accelerometers.

\section{References}

[1] Y Suzuki, A Mita : Output only estimation of inter-story drift angle for buildings using small number of accelerometers, J. Struct. Constr. Eng., AIJ, Vol.81, No.725, 1061-1070, Jul.,2016. https://doi.org/10.3130/aijs.81.1061

[2] Iwan, Wilfred D. and Moser, Michael A. and Peng, Chia-Yen: Some observations on strongmotion earthquake measurement using a digital Accelerograph, Bulletin of the Seismological Society of America (1985) 75 (5): 1225-1246

[3] G.V. Berg, G.W. Hounsner : Integral velocity and displacement of strong earthquake ground motion, Bulletin of the Seismological Society of America (1961) 51 (2): 175-189 